\title{
Contrast-enhanced ultrasound and its differential diagnosis in 21 patients with intrahepatic space-occupying lesions under the background of fatty liver
}

\author{
Yuqun Luo, Wensheng Yue, Zukun Li, Ping Wang \\ Department of Ultrasound, the Affiliated Hospital of North Sichuan Medical College, Nanchong, China \\ Contributions: (I) Conception and design: Y Luo, P Wang; (II) Administrative support: W Yue; (III) Provision of study materials or patients: Y Luo, \\ W Yue, Z Li; (IV) Collection and assembly of data: All authors; (V) Data analysis and interpretation: Y Luo, Z Li, P Wang; (VI) Manuscript writing: \\ All authors; (VII) Final approval of manuscript: All authors. \\ Correspondence to: Ping Wang. Department of Ultrasound, the Affiliated Hospital of North Sichuan Medical College, 63 Wenhua Road, Shunqing \\ District, Nanchong 637000, China. Email: wangping321wp@126.com.
}

Background The sonographic appearance in intrahepatic space-occupying lesions under the background of fatty liver is atypical. This study aimed to explore the value of contrast-enhanced ultrasonography (CEUS) in the differential diagnosis of intrahepatic space-occupying lesions under the background of fatty liver.

Methods: Twenty-one patients with intrahepatic space-occupying lesions under the background of fatty liver who were treated in our hospital from January 2017 to September 2020 and met the inclusion/ exclusion criteria were enrolled in this study. The clinical and imaging data, size, location, shape, and other characteristics of the lesions were analyzed. The accuracy of conventional ultrasound and contrast-enhanced ultrasound in diagnosing intrahepatic space-occupying lesions under the background of fatty liver were compared.

Results: The 21 patients had moderate to severe fatty liver, including 11 cases with malignant lesions (52.38\%) and 10 cases with benign lesions (47.62\%). The accuracy of CEUS (90.48\%) was significantly higher than that of conventional ultrasound $(61.90 \%)$ in diagnosing intrahepatic space-occupying lesions under the background of fatty liver. A total of 33 lesions were detected in 21 patients, which were hypoechoic. Liver abscesses mainly showed ring-shaped enhancement in the arterial phase and low enhancement in the portal phase, with abscess necrosis in the center and no significant enhancement. Liver cyst lesions showed no significant enhancement in the arterial, portal, and delayed phases. The three stages of focal fat loss were synchronized and simultaneously enhanced with surrounding fatty liver tissues. The area under the diagnostic curve of conventional ultrasound and CEUS was 0.668 and 0.809 , respectively, and the area under the curve of CEUS detection was the largest $(\mathrm{P}<0.05)$.

Conclusions: CEUS can effectively improve the diagnostic accuracy of intrahepatic space-occupying lesions under the background of fatty liver, and is an effective means to differentially diagnose intrahepatic space-occupying lesions under the background of fatty liver.

Keywords: Contrast-enhanced ultrasound; intrahepatic space-occupying lesions under the background of fatty liver; imaging signs; differential diagnosis

Submitted Dec 17, 2020. Accepted for publication Feb 05, 2021.

doi: 10.21037/apm-21-67

View this article at: http://dx.doi.org/10.21037/apm-21-67 


\section{Introduction}

Fatty liver is characterized by excessive accumulation of fat in liver cells due to a number of factors. In recent years, the incidence of fatty liver has gradually increased (1). The intrahepatic space-occupying lesions refers to the presence of one or several lesions or masses in the liver that occupy a certain volume. At present, the etiology of intrahepatic space-occupying lesions is not very clear, and it is believed that it may be related to genetic, environmental, diet and other factors. Ultrasonography has become the preferred imaging method for the clinical diagnosis of liver fat as it offers advantages such as being a simple procedure, low cost, and no radioactivity (2). In routine ultrasound examination, fatty liver shows specific signs of near-field echo enhancement and far-field echo reduction, which has a relatively high diagnostic accuracy rate (3). However, conventional ultrasonography is susceptible to interference by factors such as gas, obesity, acoustic window, and has low diagnostic specificity, which is prone to missed diagnosis, especially for the diagnosis of intrahepatic space-occupying lesions under the background of fatty liver (4). As a kind of microvascular imaging technique, contrast-enhanced ultrasonography (CEUS) can evaluate the microvascular distribution of liver tissue from the perspective of microcirculation perfusion, and differentially diagnose the characteristics of focal liver lesions, the value of which in the diagnosis of focal liver lesions has been recognized. However, reports on CEUS differential diagnosis of intrahepatic space-occupying lesions under the background of fatty liver are rare at home and abroad $(5,6)$. Therefore, this study analyzed the contrast-enhanced ultrasound characteristics of patients with intrahepatic space-occupying lesions under the background of fatty liver, and compared imaging signs of CEUS with pathological results to explore the value of CEUS in the differential diagnosis of intrahepatic space-occupying lesions under the background of fatty liver. The results presented in this study may provide a reliable basis for early clinical treatment of fatty liver.

We present the following article in accordance with the STARD reporting checklist (available at http://dx.doi. org/10.21037/apm-21-67).

\section{Methods}

\section{General information}

A total of 21 patients admitted to our hospital from January
2017 to September 2020 with intrahepatic space-occupying lesions under the background of fatty liver were enrolled as research subjects after screening according to the unified inclusion and exclusion criteria of this study. The inclusion criteria were as follows: (I) patients with no other malignant tumors; (II) patients who had not received chemotherapy, radiotherapy, or other treatments before surgery; (III) patients with complete imaging and pathological data; and (IV) patients who had undergone surgery or pathological needle biopsy diagnosis under ultrasound guidance. The exclusion criteria were as follows: (I) pregnant women during pregnancy or breastfeeding; (II) patients with mental illness or impaired consciousness; (III) patients with abnormal liver and kidney function; and (IV) patients with severe hypertension, diabetes, and coronary heart disease. Among the 21 included patients, 16 were males and five were females, aged from 31 to 66 years, with an average age of $44.69 \pm 2.55$ years. The diameter of the lesions in these patients measured $1.15-7.75 \mathrm{~cm}$. All procedures performed in this study involving human participants were in accordance with the Declaration of Helsinki (as revised in 2013). This study was approved by the Affiliated Hospital of North Sichuan Medical College [No. 2019ER (A) 024]. Informed consent was taken from all the patients.

\section{CEUS inspection method}

CEUS inspection was performed using the GE Vivid E9 color Doppler ultrasound system (GE, USA), with patients being placed in the supine position. Firstly, twodimensional ultrasound was used to observe and record the general condition of the liver through horizontal, vertical, and oblique multi-directional observation to determine the location of the lesion. Next, $1.5 \mathrm{~mL}$ of contrast agent was injected through a peripheral vein to observe the lesion in ultrasound contrast mode and record relevant data. The contrast-enhanced ultrasound process was divided into three phases: an arterial phase (5-30 s), a portal phase (31-120 s), and a delayed phase (121-360 s). After injection of the contrast agent, a timer was started immediately to record the start of enhancement time, the peak time, and the fade time.

\section{Observation indicators}

Ultrasound images were read by two experienced doctors with a senior professional title, or with a title of associate chief physician or above, using a double-blind method 
to analyze the size, location, and shape of the lesions. All patients underwent surgical treatment, and intraoperative or postoperative pathological results were used as the gold standard. The accuracy of conventional ultrasound and CEUS were subsequently compared in terms of diagnosing intrahepatic space-occupying lesions under the background of fatty liver.

\section{Statistical methods}

The data in this study were statistically analyzed by SPSS 22.0 software (IBM, New York, USA). The measurement data were described as mean \pm standard deviation $(\bar{x} \pm s)$, and the count data were described as pass rate or composition ratio, and were analyzed using the chi-squared $\left(\chi^{2}\right)$ test. The receiver operating characteristic (ROC) curve was used to analyze the diagnostic efficiency of conventional ultrasound and CEUS for intrahepatic space-occupying lesions under the background of fatty liver. Results with $\mathrm{P}<0.05$ were considered to be statistically significant.

Table 1 Clinical data

\begin{tabular}{lcc}
\hline Lesions & Case $(\mathrm{n})$ & Proportion (\%) \\
\hline Primary liver cancer & 7 & 33.33 \\
Metastatic liver cancer & 4 & 26.67 \\
Hepatic hemangioma & 5 & 23.81 \\
Hepatapostema & 1 & 4.76 \\
Liver cyst & 1 & 4.76 \\
Focal fat loss & 3 & 14.29 \\
\hline
\end{tabular}

\section{Results}

\section{Clinical data of the patients}

All 21 patients suffered from moderate to severe fatty liver. Among them, 11 cases had malignant lesions (52.38\%) and 10 cases had benign lesions (47.62\%). The specific pathological types are shown in Table 1.

\section{The value of conventional ultrasound and CEUS in diagnosing intrabepatic space-occupying lesions under the background of fatty liver}

The accuracy of conventional ultrasound and CEUS in diagnosing intrahepatic space-occupying lesions under the background of fatty liver was $61.90 \%$ and $90.48 \%$, respectively, and the difference between the two inspection methods was statistically significant $(\mathrm{P}<0.05$, Table 2$)$.

\section{Imaging features}

A total of 33 lesions were detected in the 21 included patients, which were hypoechoic. Among them, the enhancement characteristics of primary liver cancer lesions were mainly manifested as typical "fast-in and fast-out" characteristics that the rapid enhancement of arterial phase was more obvious than that of the surrounding normal liver tissues. The degree of tumor enhancement in portal and delayed stage decreased rapidly and was lower than that in normal liver tissues (Figure 1). Metastatic liver cancer lesions began to enhance rapidly from the periphery in the arterial phase and showed a ring shape, and were quickly cleared in the portal and delayed phases. One case of metastatic

Table 2 Diagnostic accuracy of intrahepatic space-occupying lesions in the diagnosis of fatty liver by conventional ultrasound and CEUS [n (\%)]

\begin{tabular}{|c|c|c|c|c|c|c|c|}
\hline $\begin{array}{l}\text { Inspection } \\
\text { method }\end{array}$ & $\begin{array}{c}\text { Primary liver } \\
\text { cancer }\end{array}$ & $\begin{array}{c}\text { Metastatic liver } \\
\text { cancer }\end{array}$ & $\begin{array}{c}\text { Hepatic } \\
\text { hemangioma }\end{array}$ & Hepatapostema & Liver cyst & Focal fat loss & Sum \\
\hline $\begin{array}{l}\text { Conventional } \\
\text { ultrasound }\end{array}$ & $4(57.14)$ & $3(75.00)$ & $3(60.00)$ & $1(25.00)$ & $0(0.00)$ & $2(50.00)$ & $13(61.90)$ \\
\hline CEUS & $6(85.71)$ & $4(100.00)$ & $4(80.00)$ & $4(100.00)$ & $4(100.00)$ & $4(100.00)$ & $19(90.48)$ \\
\hline$\chi^{2}$ & - & - & - & - & - & - & 4.725 \\
\hline
\end{tabular}

CEUS, contrast-enhanced ultrasonography. 

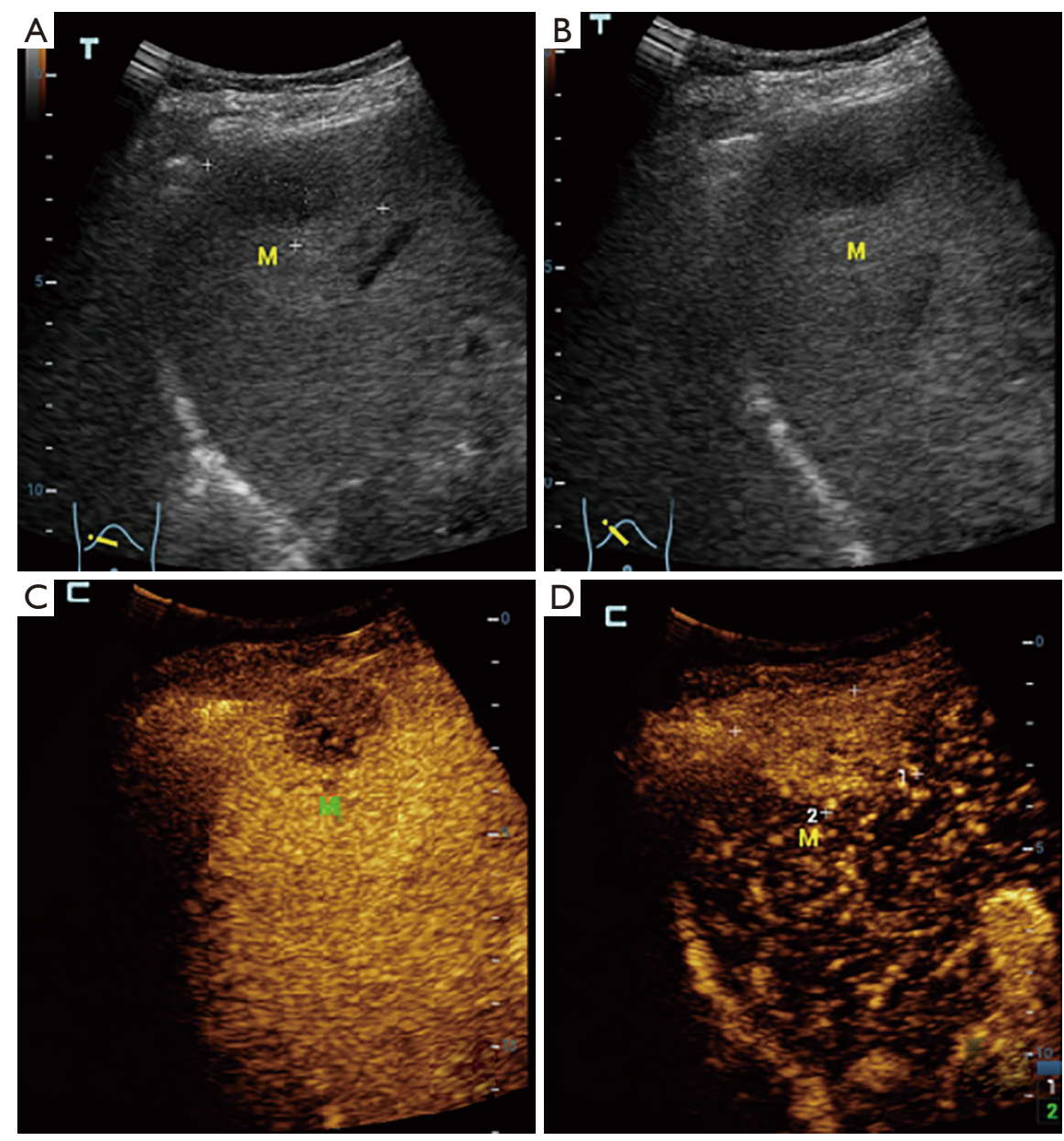

Figure 1 Images of primary liver cancer. (A,B) Conventional ultrasound showed diffuse enhancement of the liver parenchyma echo, attenuation of the deep echo, and $3.3 \times 2.6 \mathrm{~cm}$ hypoecho under the capsule of the upper right anterior lobe, with a regular morphology and clear boundary; (C) enhanced ultrasound showed high enhancement and an increased measured value in the arterial phase of the hypoechoic lesion in the upper right anterior lobe; (D) enhanced ultrasound showed early low enhancement of the portal vein of the hypoechoic lesion in the upper right anterior lobe, manifested as the typical "fast-in and fast-out" characteristics.

liver carcinoma showed uniform high enhancement in the arterial phase. Hepatic hemangioma lesions primarily displayed peripheral enhancement, which were nodular. There were two cases with uneven centripetal filling and two cases with uniform centripetal filling, which showed high enhancement in the portal and delayed phases, and gradually decreased in the delayed phase to a slightly higher total enhancement. Liver abscesses primarily displayed ring-shaped enhancement in the arterial phase, and showed low enhancement in the portal phase, with abscess necrosis in the center and no significant enhancement. Liver cyst lesions showed no significant enhancement in the arterial, portal, and delayed phases (Figure 2). The three stages of focal fat loss were simultaneously and equally enhanced with the surrounding fatty liver tissue. The enhanced characteristics of CEUS in the 21 patients with hepatic space-occupying lesions under the background of fatty liver are shown in Table 3.

\section{Analysis of the diagnostic efficacy of conventional ultrasound and CEUS in the diagnosis of intrahepatic space-occupying lesions under the background of fatty liver}

The ROC curve was used to analyze the diagnostic efficacy of conventional ultrasound and CEUS in the diagnosis of intrahepatic space-occupying lesions under the background 

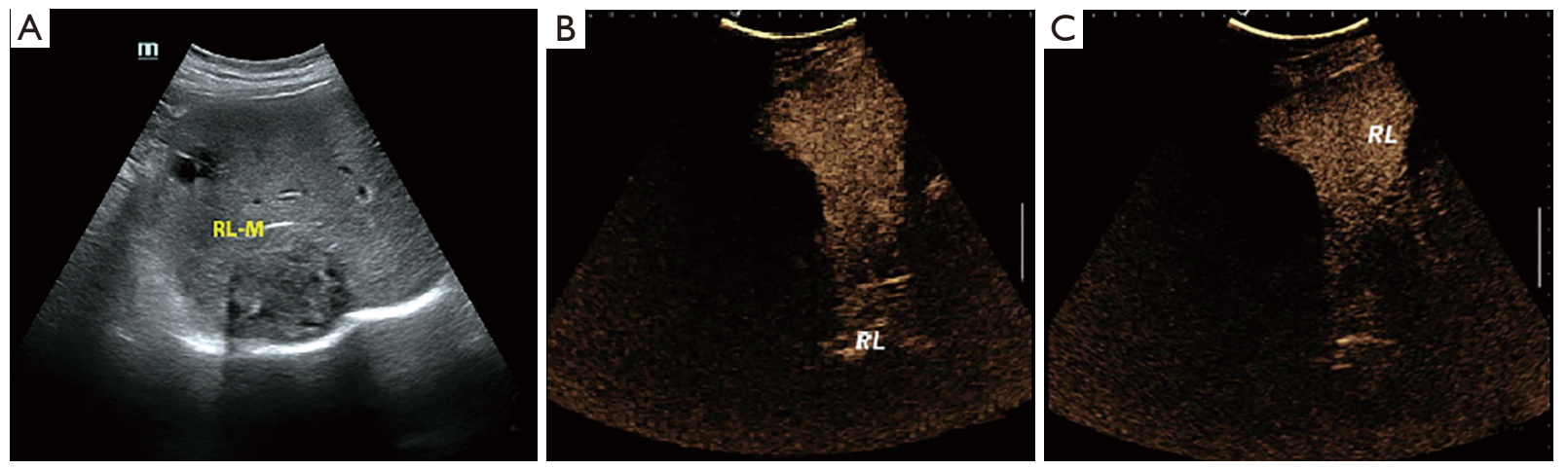

Figure 2 Images of multiple hepatic cysts. (A) Conventional ultrasound showed heterogeneous hypoechoic lesions in the upper right posterior lobe; (B,C) enhanced ultrasound showed no enhancement in the arterial and portal phases of the heterogeneous hypoechoic lesion in the upper right posterior lobe.

Table 3 Enhanced characteristics of CEUS in 21 cases of intrahepatic space-occupying lesions under the background of fatty liver

\begin{tabular}{llll}
\hline \multirow{2}{*}{ Lesions } & \multicolumn{2}{c}{ Enhancement characteristics of lesions } \\
\cline { 2 - 3 } & \multicolumn{1}{c}{ Arterial phase } & Portal phase & Delayed phase \\
\hline Primary liver cancer & Uniform or uneven high enhancement & Low or equal enhancement & Low enhancement \\
Metastatic liver cancer & High enhancement, annular & Low enhanced & Low enhancement \\
Hepatic hemangioma & Annular or overall high enhancement & High enhancement & Slightly high enhancement \\
Hepatapostema & Irregular ring with high enhancement, no & Peripheral enhancement, no & Peripheral low enhancement and \\
& obvious enhancement in the center & obvious central enhancement & central no obvious enhancement \\
Liver cyst & No obvious enhancement & No obvious enhancement & No obvious enhancement \\
Focal fat loss & Equal enhancement & Equal enhancement & Equal enhancement \\
\hline
\end{tabular}

CEUS, contrast-enhanced ultrasonography.

Table 4 Diagnostic efficacy of conventional ultrasound and CEUS in intrahepatic space-occupying lesions under the background of fatty liver

\begin{tabular}{|c|c|c|c|c|c|}
\hline Predictive factor & Optimal threshold & Sensitivity & Specificity & AUC of ROC curve & $95 \% \mathrm{Cl}$ \\
\hline CEUS & 19.63 & 0.973 & 0.803 & 0.809 & $0.600-1.000$ \\
\hline
\end{tabular}

CEUS, contrast-enhanced ultrasonography; AUC, area under the curve; ROC, receiver operating characteristic; $\mathrm{Cl}$, confidence interval.

of fatty liver. The area under the diagnostic ROC curve of conventional ultrasound and CEUS was 0.668 and 0.809 , respectively. The area under each curve of CEUS inspection was the largest (Table 4 and Figure 3).

\section{Discussion}

With the continuous development of ultrasound examination technology and the accumulation of diagnostic experience, conventional ultrasound has a high diagnostic accuracy for normal liver nodules based on the size, shape, echo, and color blood flow information of the lesion, and can provide a reliable basis for its pathological diagnosis $(7,8)$. As the living standards of people improve, the incidence of fatty liver is also increasing annually. However, the sonographic appearance of intrahepatic space-occupying lesions under the background of fatty liver is atypical, and conventional ultrasound examinations cannot display the microvessels in the lesions. At the same time, due to the interference of fatty liver, the original blood flow signal 


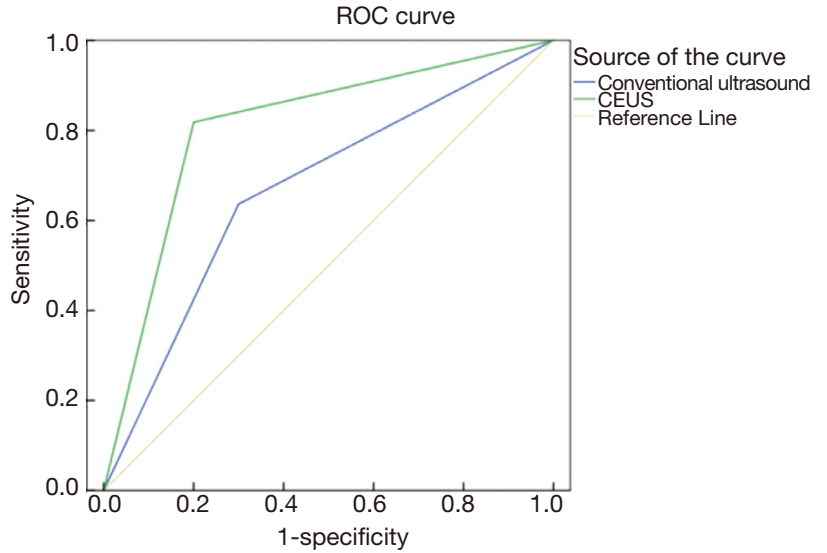

Figure 3 Curve analysis of intrahepatic space-occupying lesions under the background of fatty liver by conventional ultrasound and CEUS. ROC, receiver operating characteristic; CEUS, contrastenhanced ultrasonography.

in the lesion is weakened or even disappeared, resulting in decreased accuracy of diagnosis of intrahepatic spaceoccupying lesions under the background of fatty liver, thereby leading to missed diagnoses and misdiagnoses $(9,10)$.

At present, CEUS is the main clinical imaging method for evaluating liver blood flow and diagnosing focal liver lesions. As a novel examination method developed on the basis of conventional ultrasound, CEUS has the advantages of simple operation, safety, low cost and repeatability, with the advantageous in reflecting the blood supply compared with CT and MRI, CEUS, which can provide clinicians with information about the distribution of blood vessels and blood flow (11). Compared with benign lesions, malignant lesions have significantly more blood vessels, a richer blood supply, and more irregular shapes. Therefore, CEUS plays an important role in the differential diagnosis of benign and malignant lesions (12). As a product of the third revolution of ultrasound imaging, CEUS can enhance the blood flow scattering signal of the human body via intravenous injection of ultrasound contrast agent, which will allow for dynamic observation of the microvascular perfusion information of the tissue in real time (13). In addition, by injecting ultrasound contrast agents, it is not only possible to observe the distribution of lesions and small blood vessels in the liver parenchyma that cannot be displayed by color Doppler in real time, but also to distinguish the microperfusion characteristics of normal liver parenchyma and liver lesions, which provides unique advantages for the diagnosis of liver lesions $(14,15)$. In this study, the results demonstrated that the accuracy of CEUS in the diagnosis of intrahepatic space-occupying lesions under the background of fatty liver was $90.48 \%$, which was significantly higher than that of conventional ultrasound.

CEUS can perform arterial, portal, and delayed phase scans. Malignant lesions usually show low enhancement in the portal and parenchymal phases, while benign lesions often display equal or high enhancement (16). The underlying mechanism for this is primarily related to blood sinusoid aggregation and the reticuloendothelial system. In this study, we investigated CEUS in the diagnosis of malignant lesions to show that primary liver cancer was mainly characterized by "fast-in and fast-out" enhancement. The contrast agent filled rapidly and uniformly in arterial phase lesions, and perforator angiography could be seen in some lesions. There were areas of hemorrhage and necrosis in the larger lesions, as well as uneven areas without enhancement. The signs of contrast-enhanced ultrasound in the primary lesions of metastatic liver cancer were different, and could be divided into ring enhancement, mass enhancement, delayed enhancement, and no enhancement according to the differences in arterial phase enhancement. In this study, there was one patient with liver metastases that had gastric cancer as the primary foci, and contrastenhanced ultrasound displayed the "fast-in and fast-out" characteristic, which may be related to the small lesions and abundant blood supply. Sidorov et al. (17) reported that CEUS had good consistency with enhanced CT in the diagnosis of intrahepatic space-occupying lesions under the background of fatty liver. However, some lesions, which lack contrast-enhanced ultrasound signs due to a larger volume or insufficient blood supply, still need to be diagnosed in conjunction with other clinical examinations and medical history.

Hepatic hemangioma lesions mainly displayed peripheral nodular enhancement, uniform or uneven centripetal filling, and high enhancement in the portal and delayed phases. In this study, two patients showed high enhancement in the portal phase, which decreased to overall equal enhancement in the delayed phase. This may be related to the slow clearance of severe fatty liver tissue. Liver cells swell during steatosis and the sinusoids are compressed, resulting in slow portal blood flow. Contrast-enhanced ultrasound of liver abscesses mainly displayed circular enhancement in the arterial phase, low enhancement in portal clearance, and no enhancement in the central abscess necrosis. Abscesses have different contrast-enhanced ultrasound signs in different periods. When new internal blood vessels are formed, 
overall high enhancement is seen in the arterial phase, which needs to be distinguished from primary liver cancer with central necrosis by comprehensive analysis combined with tumor biomarkers as well as clinical symptoms and signs $(18,19)$. Liver cysts are easier to distinguish than other types of lesions, and there is no obvious enhancement in the three stages. Focal fat loss in the liver is also called the "liver island", some lesions of which are prone to be misdiagnosed as substantial space-occupying due to the regular shape. CEUS of liver cysts is primarily manifested as enhancement in synchronization with the surrounding liver parenchyma without significant space-occupying effect. Amadori et al. pointed out that CEUS can accurately diagnose the "liver island", which helps to differentiate focal fat loss from liver cysts, liver hemangioma, and some other lesions (20). In addition, there are some shortcomings in this study, such as small sample size and single-center study, therefore further expansion of sample size and multi-center study is needed in the future.

In summary, CEUS can effectively improve the diagnostic accuracy of intrahepatic space-occupying lesions under the background of fatty liver, and is an effective means for differential diagnosis of intrahepatic spaceoccupying lesions under the background of fatty liver.

\section{Acknowledgments}

Funding: None.

\section{Footnote}

Reporting Checklist: The authors have completed the STARD reporting checklist. Available at http://dx.doi.org/10.21037/ apm-21-67

Data Sharing Statement: Available at http://dx.doi. org/10.21037/apm-21-67

Conflicts of Interest: All authors have completed the ICMJE uniform disclosure form (available at http://dx.doi. org/10.21037/apm-21-67). The authors have no conflicts of interest to declare.

Ethical Statement: The authors are accountable for all aspects of the work in ensuring that questions related to the accuracy or integrity of any part of the work are appropriately investigated and resolved. This study was approved by the Affiliated Hospital of North Sichuan
Medical College [No. 2019ER (A) 024]. All procedures performed in this study involving human participants were in accordance with the Declaration of Helsinki (as revised in 2013). Informed consent was taken from all the patients.

Open Access Statement: This is an Open Access article distributed in accordance with the Creative Commons Attribution-NonCommercial-NoDerivs 4.0 International License (CC BY-NC-ND 4.0), which permits the noncommercial replication and distribution of the article with the strict proviso that no changes or edits are made and the original work is properly cited (including links to both the formal publication through the relevant DOI and the license). See: https://creativecommons.org/licenses/by-nc-nd/4.0/.

\section{References}

1. Hui SCN, So HK, Chan DFY, et al. Validation of waterfat MRI and proton MRS in assessment of hepatic fat and the heterogeneous distribution of hepatic fat and iron in subjects with non-alcoholic fatty liver disease. Eur J Radiol 2018;107:7-13.

2. Sharma K, Gupta N, Goyal K, et al. Evaluation of polymerase chain reaction in space-occupying lesions of liver reported as granulomatous inflammation/tuberculosis on fine-needle aspiration cytology. Cytojournal 2017;14:1 .

3. Bessone F, Dirchwolf M, Rodil MA, et al. Review article: drug-induced liver injury in the context of nonalcoholic fatty liver disease - a physiopathological and clinical integrated view. Aliment Pharmacol Ther 2018;48:892-913.

4. Amit S, David, JP, Dominik B, et al. FRI-504-Sorafenib use in patients with HCC on a background of nonalcoholic fatty liver disease. J Hepatol 2019;70:e621.

5. Mathada UT, Svinarenko M, Bushart N, et al. Impact of Trp53 on tumor heterogeneity in the background of liver fibrosis. J Hepatol 2018;68:S686.

6. Kanwal F, Kramer JR, Li L, et al. Effect of Metabolic Traits on the Risk of Cirrhosis and Hepatocellular Cancer in Nonalcoholic Fatty Liver Disease. Hepatology 2020;71:808-19.

7. Munteanu M, Pais R, Peta V, et al. Long-term prognostic value of the FibroTest in patients with non-alcoholic fatty liver disease, compared to chronic hepatitis C, B, and alcoholic liver disease. Aliment Pharmacol Ther 2018;48:1117-27.

8. Mozes FE, Tunnicliffe EM, Moolla A, et al. Mapping tissue water T1 in the liver using the MOLLI T1 method 
in the presence of fat, iron and B0 inhomogeneity. NMR Biomed 2019;32:e4030.

9. Mathada UT, Svinarenko M, Bushart N, et al. Impact of Trp53 on tumor heterogeneity in the background of liver fibrosis. J Hepatol 2018;68:S686.

10. Chen XP, Liu J, Zhou J, et al. Combination of CEUS and MRI for the diagnosis of periampullary space-occupying lesions: a retrospective analysis. BMC Med Imaging 2019;19:77.

11. Cocciolillo S, Tavani R, Di Berardino M, et al. Evaluation of Intraparenchymal and Portal Blood Flow by Contrast Enhanced Ultrasound (CEUS) and Liver Stiffness by Fibroscan in Nonalcoholic Fatty Liver Disease (NAFLD) and Chronic Hepatitis C (CHC). Gastroenterology 2017;152:S215.

12. Ikeda A, Oiwa Y, Kokuryu H. Contrast-enhanced ultrasonography evaluation of hepatocellular carcinoma with peritumoral fat-spared area: a case report. J Med Ultrason (2001) 2018;45:325-9.

13. Kushner T, Tholey D, Dodge J, et al. Outcomes of liver transplantation for acute fatty liver disease of pregnancy. Am J Transplant 2019;19:2101-7.

14. Breher-Esch S, Sahini N, Trincone A, et al. Genomics of lipid-laden human hepatocyte cultures enables drug target screening for the treatment of non-alcoholic fatty liver

Cite this article as: Luo Y, Yue W, Li Z, Wang P. Contrastenhanced ultrasound and its differential diagnosis in 21 patients with intrahepatic space-occupying lesions under the background of fatty liver. Ann Palliat Med 2021;10(3):3097-3104. doi: 10.21037/apm-21-67 disease. BMC Med Genomics 2018;111:1234-5.

15. George J, Ray B. FRI-466-A single centre experience of transarterial radioembolisation using Rhenium 188 for treatment of liver space occupying lesions. J Hepatol 2019;70:e602.

16. Wu CN, Duan SF, Mu XT, et al. Assessment of optic nerve and optic tract alterations in patients with orbital space-occupying lesions using probabilistic diffusion tractography. Int J Ophthalmol 2019;12:1304-10.

17. Sidorov D, Petrov L, Lozhkin M, et al. Portal vein ligation vs ALPPS for colorectal metastatic liver cancer. Eur J Cancer 2017;72:S64-4.

18. Schwarze V, Marschner C, Völckers W, et al. Diagnostic value of contrast-enhanced ultrasound versus computed tomography for hepatocellular carcinoma: a retrospective, single-center evaluation of 234 patients. J Int Med Res 2020;48:300060520930151.

19. Nio K, Yamashita T, Kaneko S. The evolving concept of liver cancer stem cells. Mol Cancer. 2017;16:4.

20. Amadori M, Barone D, Scarpi E, et al. Dynamic contrastenhanced ultrasonography (D-CEUS) for the early prediction of bevacizumab efficacy in patients with metastatic colorectal cancer. Eur Radiol 2018; 28:2969-78.

(English Language Editor: A. Kassem) 